\title{
THE INNER M1-67 NEBULA: CORONAGRAPHIC IMAGING AND ECHELLE OBSERVATIONS
}

\author{
A. NOTA ${ }^{*}$ M. CLAMPIN , M. SIRIANNI ** and P. GREENFIELD \\ Space Telescope Science Institute, Baltimore, MD, U.S.A. \\ and \\ D.A. GOLIMOWSKI \\ The Johns Hopkins University, Baltimore, MD, U.S.A.
}

\begin{abstract}
Coronagraphic and spectroscopic observations of the nebula M1-67 surrounding WR124 reveal an axisymmetric outflow.
\end{abstract}

\section{Introduction}

M 1-67 is a bright, clumpy nebula surrounding WR124, a Population I WN8 star characterized by a very high velocity of $200 \mathrm{~km} / \mathrm{s}$, similar to that of the nebula (Bertola 1964; Cohen \& Barlow 1975). Although first classified as a planetary nebula (Perek \& Kohutek 1967), M1-67's classification remains the subject of debate (Pismis \& Recillaz-Cruz 1979; van der Hucht et al. 1985). Recent abundance analysis of the nebula (Esteban et al. 1991, 1993) revealed a $\mathrm{N}$ enhancement and $\mathrm{O}$ deficiency, suggesting material ejected from the star itself in a previous, maybe LBV, phase. The dynamics of the nebula are also the subject of debate: from Fabry-Perot interferometry in $\mathrm{H} \alpha$, Pismis \& Recillas-Cruz (1979) found several components in the velocity field of the nebula and proposed anisotropic mass loss from the star. Later, employing a similar technique with improved resolution, Chu \& Treffers (1981) suggested that the velocity components observed were probably due to separate "outbursts" experienced by the star at different epochs. Alternatively, Solf \& Carsenty (1982) explained their high resolution long-slit spectroscopy results with the presence of a thin expanding shell, probably produced by the interaction of the strong stellar wind with pre-existing material lost by the star in a previous phase.

\section{The observations}

We have observed M1-67 with the Johns Hopkins University (JHU) Adaptive Optics Coronagraph (AOC) at the Swope 40" telescope (Las Campanas, Chile), on 1990 June 19. Images were taken in the light of $\mathrm{H} \alpha+[\mathrm{NII}]$

* Affiliated to ESA, Astrophysics Division, Space Science Department of ESA

** Istituto di Astronomia, Padova 
$(\lambda=6560 \AA, \Delta \lambda=110 \AA)$. The field of view is $42^{\prime \prime} \times 63^{\prime \prime}$, with a plate scale of $0^{\prime \prime} .22$ / pixel. A circular occulting mask of $5^{\prime \prime}$ in diameter was used to block the light from WR124. The image is shown in Figure 1a. The inner M1-67 nebula appears very clumpy and remarkably axisymmetric. All the emission is in the forms of blobs, 2-12 arcseconds in size, concentrated mainly within two cones originating from the star at a position angle $\sim 0^{\circ}$. The overall bipolar symmetry is striking and now clearly visible due to the high contrast achieved by the coronagraph in the inner regions: this prompted us to reinvestigate the dynamics of the nebula and, in particular, to perform dynamical mapping of the individual blobs we identified in the coronagraphic images, which had been mostly missed by the previous investigations (Solf \& Carsenty 1982; Chu \& Treffers 1981).
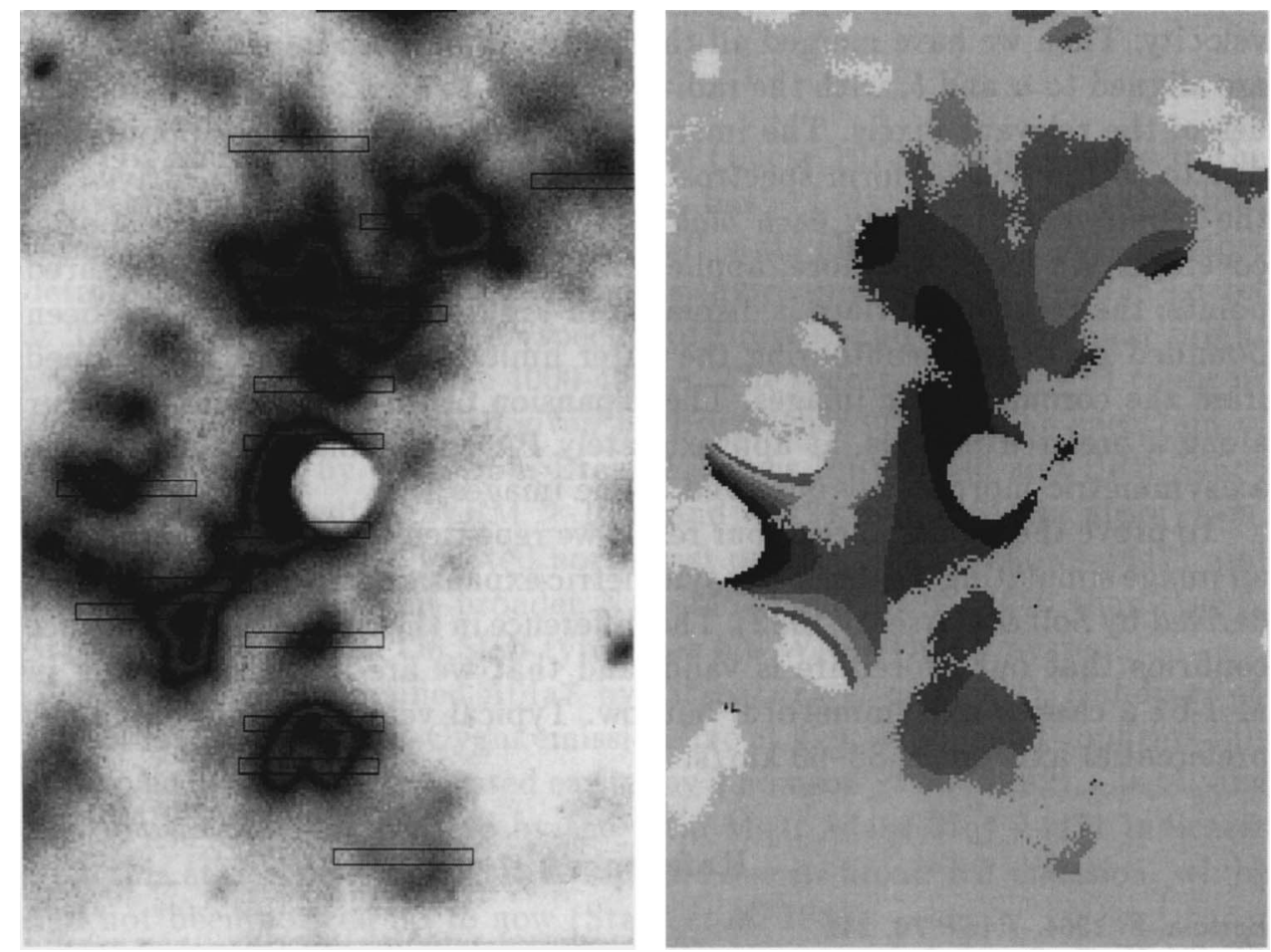

Fig. 1. a) left: Coronagraphic image of the M1-67 nebula (Ho+[NII]). The slit locations $\left(1^{\prime \prime} \times 9^{\prime \prime}\right)$ of the spectroscopic observations are marked. b) right: radial velocity interpolated surface of the M1-67 nebula. Notice the axial symmetry in the velocity distribution.

Echelle spectra of the M1-67 nebula were obtained in $1990 \mathrm{July}$ at the 3.6m telescope at ESO, La Silla, with EFOSC configured in an echellette mode to achieve a spectral resolution of $1.1 \AA /$ pixel at $6584 \AA$. A slit of $1^{\prime \prime} \times 9^{\prime \prime}$ (with a spatial scale of $0^{\prime \prime} .337 /$ pixel), was used to take moderate 
resolution spectra of all the individual blobs we identified in the images. In total, we obtained spectra of 17 blobs, in addition to several spectra of the central star, which was used as starting position for our pointing offsets. In Fig. 1a we have superposed the slit positions on the coronagraphic image, so that it is possible to visualize our spectroscopic coverage of the nebula. We believe our slit pointing error is lower than $1^{\prime \prime}$.

\section{Results}

Radial velocities have been computed, for each slit position, from the [NII] $6584 \AA$ line profile. Our overall result is presented in Figure $1 \mathrm{~b}$, where we have attempted to condense in one figure the radial velocities of the individual blobs while maintaining the spatial information of the blobs within the nebula. First, we have assigned to each point along each slit its radial velocity. Then we have merged all this information in an image, whose axes are aligned to $\alpha$ and $\delta$, with the radial velocities written as the intensity values of the relevant pixels. The image so constructed is sparsely populated, due to to the non uniform spectroscopic coverage of the nebula, driven by the requirement to study each blob rather than obtaining uniform spatial coverage. We have, therefore, applied a 2-D interpolation to the measured points: the resulting surface is displayed in Figure 1b. The surface has been bounded by a mask contouring the outer limits of the nebula as obtained from the coronagraphic images. The expansion of the blobs seems to occur along a preferential axis, at approximately $\mathrm{PA} \simeq 0^{\circ}$, consistently with the axisymmetric morphology observed in the images.

To prove the significance of our result we repeated the same procedure on an image simulating a spherically symmetric expansion (with the parameters derived by Solf \& Carsenty 1982). The difference in the corresponding surface confirms that our procedure is valid, and that we are indeed observing in M 1-67 a case of axisymmetrical outflow. Typical velocity values along the preferential axis are $\simeq 35-60 \mathrm{~km} / \mathrm{s}$.

\section{References}

Bertola, F. 1964, PASP 76, 241

Chu, Y.-H., Treffers, R.R. 1981, ApJ 249, 586

Cohen, M., Barlow, M.J. 1975, Astrophys. Letters 16, 165

Esteban, C., Smith, L.J., Vilchez, J.M., Clegg, R.E.S. 1991, $A \& A$ 244, 205

Esteban, C., Smith, L.J., Vilchez, J.M., Clegg, R.E.S. 1993, $A \& A$ 272, 299

Perek, L., Kohutek, L. 1967, Catalog of Galactic Planetary Nebulae, (Prague: Czechoslovak Institute of Science).

Pismis, P., Recillaz-Cruz, E. 1979, Rev. Mexicana Astron. Astrof. 4, 271

Solf, J., Carsenty, U. 1982, $A \& A$ 116, 54

van der Hucht, K.A., Jurriens, T.A., Olnon, F.M., Thé, P.S., Wesselius, P.R., Williams, P.M. 1985, $A \& A$ (Letters) 145, L13 